\title{
Associated Higgs Boson Production with heavy Quarks in e+e- Collisions: SUSY-QCD
}

\author{
Petra Häfliger ${ }^{1,2 *}$ and Michael Spira ${ }^{2}$ \\ ${ }^{1}$ Institute for Particle Physics, ETH Zürich, CH-8093 Zürich, Switzerland \\ ${ }^{2}$ Paul Scherrer Institute, CH-5232 Villigen PSI, Switzerland \\ E-mail: petra.haefliger@phys.ethz.ch, michael.spira@psi.ch
}

\begin{abstract}
The processes $e^{+} e^{-} \rightarrow t \bar{t} / b \bar{b}+$ Higgs allow to measure the Yukawa couplings between Higgs bosons and heavy quarks in supersymmetric theories. The complete set of next-to-leading order SUSY-QCD corrections to the cross sections of these processes have been determined in the minimal supersymmetric extension of the Standard Model. They turn out to be $\mathscr{O}(10-20 \%)$ and thus important for future linear $e^{+} e^{-}$colliders
\end{abstract}

International Europhysics Conference on High Energy Physics

July 21st - 27th 2005

Lisboa, Portugal

* Speaker. 


\section{Calculation of NLO SUSY-QCD Corrections}

The leading order (LO) Higgs radiation off heavy quarks splits into three different classes of contributions: (i) Higgs radiation off the heavy (anti)quark, (ii) Higgs radiation off the $Z$ boson (only scalar Higgs radiation) and (iii) $Z$ boson splitting into scalar-pseudoscalar Higgs pairs with one of them dissociating into a heavy $Q \bar{Q}$ pair [1]. Depending on the masses of the corresponding particles resonant contributions will arise, which require the inclusion of finite decay widths of the $Z$ and Higgs bosons in the corresponding propagators. We have used conventional Breit-Wigner propagators for the resonant $Z \rightarrow b \bar{b}$ and $\phi^{0} \rightarrow t \bar{t} / b \bar{b}$ decays as in previous analyses [2].

We include the QCD corrections of Ref. [2] with the QCD coupling $\alpha_{s}$ evaluated at NLO with 5 active flavours at the renormalisation scale $\mu_{R}=\sqrt{s}$ with $s$ being the $e^{+} e^{-}$c.m. energy squared. The bottom Yukawa couplings are computed at the scale of the corresponding Higgs-momentum flow. This choice absorbs large logarithmic contributions of the pure QCD corrections [2].

The NLO SUSY-QCD corrections arise from virtual gluino and stop/sbottom exchange (for further details see Ref. [1]). They consist of self-energy, vertex and box contributions, which are calculated within dimensional regularisation in the standard way. Since all virtual particles are massive, no infrared nor collinear singularities arise. The ultraviolet divergences are removed by the renormalisation of the quark masses and Yukawa couplings. The latter is connected to the quark mass renormalisation. In the case of $t \bar{t}+$ Higgs production the top mass has been renormalised onshell in the propagators as well as in the Yukawa couplings. The same prescription has also been chosen for the bottom mass, since the virtual gluino and sbottom masses are too large to develop large logarithmic contributions. Thus the renormalisation of the bottom quark mass is given by

$$
m_{b}^{0}=\bar{m}_{b}\left(\mu^{2}\right)[1+\underbrace{\left(-\frac{\alpha_{s}}{\pi} \Gamma(\varepsilon)(4 \pi)^{\varepsilon}\right)}_{\delta_{Q C D}}+\underbrace{\left(\frac{\tilde{\Sigma}\left(m_{b}\right)}{\bar{m}_{b}\left(\mu^{2}\right)}\right)}_{\delta_{S Q C D}}]
$$

where $m_{b}^{0}$ denotes the bare bottom mass, $\bar{m}_{b}\left(\mu^{2}\right)$ the $\overline{\mathrm{MS}}$ mass at the scale $\mu$ and $\delta_{(S) Q C D}$ the corresponding (SUSY-)QCDcounter terms. The SUSY-QCD contribution to the bottom quark selfenergy is displayed by $\tilde{\Sigma}\left(m_{b}\right)$ with on-shell momentum. This renormalisation prescription ensures that the gluino and sbottom contributions are decoupled from the running of the bottom Yukawa couplings. Thus we are left with the pure $\overline{\mathrm{MS}}$ Yukawa couplings of QCD.

The final result can be cast into the form

$$
\sigma\left(e^{+} e^{-} \rightarrow Q \bar{Q} \phi^{0}\right)=\sigma_{L O}\left(e^{+} e^{-} \rightarrow Q \bar{Q} \phi^{0}\right)\left\{1+\left[C_{Q C D}+C_{S Q C D}\right] \frac{\alpha_{S}(s)}{\pi}\right\},
$$

where $\sigma_{L O}\left(e^{+} e^{-} \rightarrow Q \bar{Q} \phi^{0}\right)$ denotes the LO cross section and $C_{(S) Q C D}$ the coefficients of the (SUSY-)QCD corrections.

For large values of $\operatorname{tg} \beta$ there are significant non-decoupling corrections to $b \bar{b} \phi^{0}$ production, which can be absorbed in the bottom Yukawa couplings in a universal way [3]. In Refs. [4, 5] it has been shown that these contributions can be resummed to improve the reliability of the perturbative result. 


\section{Results}

The numerical results will be presented for a linear $e^{+} e^{-}$collider with c.m. energy of $1 \mathrm{TeV}$. We have chosen the Snowmass point SPS5 for Higgs radiation off top quarks and SPS1b for the bottom quark case [6]. The pseudoscalar Higgs mass is left free in both scenarios in order to scan the corresponding Higgs mass ranges.

The total cross section for pseudoscalar Higgs radiation off top quarks is of $\mathscr{O}\left(10^{-2} f b\right)$ for pseudoscalar Higgs masses below about $350 \mathrm{GeV}$, while above it rapidly increases to a level of $1 \mathrm{fb}$ due to the intermediate on-shell $H \rightarrow t \bar{t}$ decay. The total size of the corrections amounts to $\mathscr{O}(10 \%)$ apart from the threshold of the resonant contribution, where the Coulomb singularity raises the QCD corrections to more than 100\% [7]. The cross section for the light scalar Higgs boson is always of the order of $1 \mathrm{fb}$ with small corrections due to the partial cancellation of QCD and SUSY-QCD corrections (see Fig. 1a) while for the heavy scalar Higgs boson it decreases down to $\mathscr{O}\left(10^{-2} f b\right)$ up to the $t \bar{t}$-threshold. The total cross sections for $b \bar{b} \phi^{0}$ production reach a size of $\mathscr{O}(10 \mathrm{fb})$ for smaller pseudoscalar masses. All the total cross section drop down towards $M_{A} \sim 500 \mathrm{GeV}$, caused by the kinematical closure of the intermediate on-shell HA pair production.
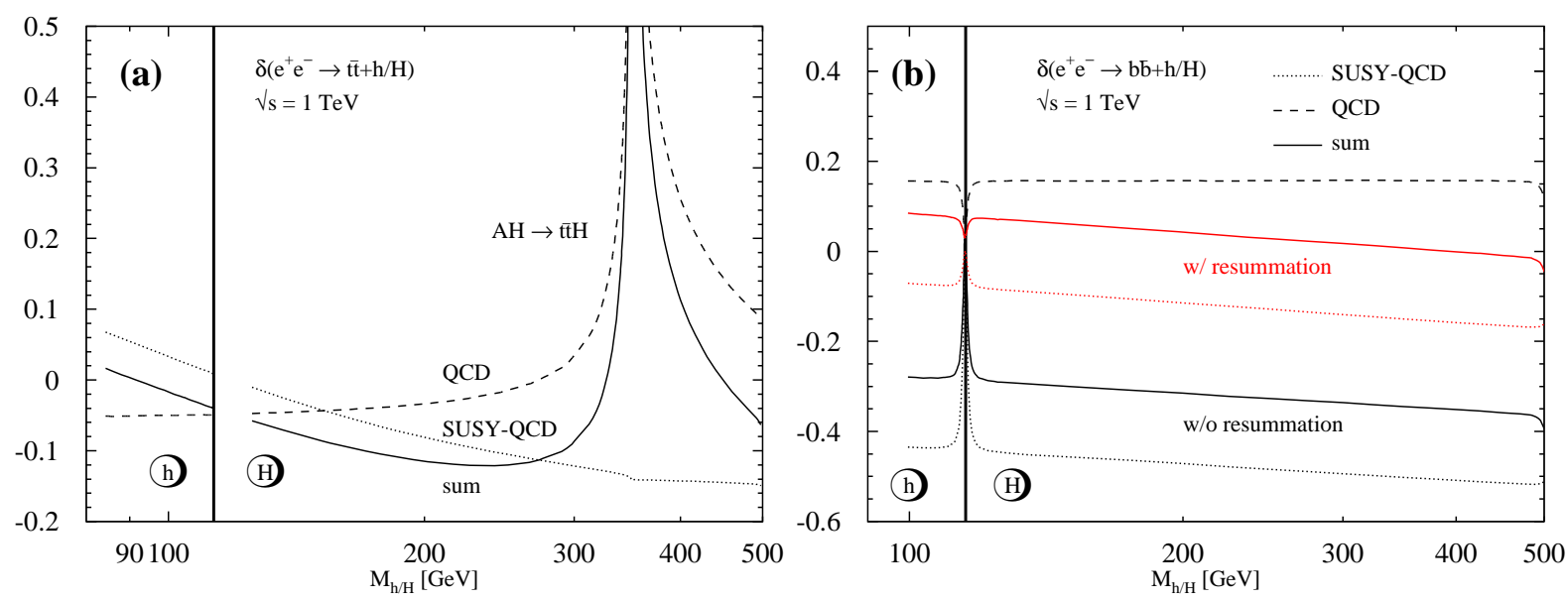

Figure 1: Relative QCD, SUSY-QCD and total corrections to scalar Higgs radiation off top quark (a) and bottom quark (b), respectively. The sharp (fi nite) peak around $M_{H}=350 \mathrm{GeV}$ originates from the Coulomb singularity in the QCD corrections to the resonant $A \rightarrow t \bar{t}$ decay.

The individual relative corrections, defined as $\sigma_{N L O}=\sigma_{L O}\left(1+\delta_{Q C D}+\delta_{S Q C D}\right)$, can be inferred from Fig. 1a for $b \bar{b}+h / H$. Except for the threshold region of the resonant part, the QCD corrections are of moderate size [2]. The QCD Coulomb singularity for $M_{A} \sim 350 \mathrm{GeV}$ is much more pronounced than in the pseudoscalar case, since for the heavy scalar Higgs boson the $S$-wave pseudoscalar Higgs decay $A \rightarrow t \bar{t}$ constitutes the resonant part. The relative threshold corrections remain finite in both cases due to the remaining continuum contributions. The SUSY-QCD corrections are of similar magnitude as the pure QCD corrections but of opposite sign. Thus, we observe a large cancellation of the QCD corrections against the SUSY-QCD part. This signalises the importance of including both types of corrections in future analyses. An analogous pictures emerges for the pseudoscalar Higgs boson in the final state. It should be noted that the SUSY-QCD corrections can 
either be constructive or destructive depending on the MSSM scenario.

The relative corrections for associated Higgs production with bottom quarks are depicted in Fig. $1 \mathrm{~b}$. The pure SUSY-QCD and total corrections are shown without and with resummation for scalar Higgs bosons. It is clearly visible that the resummed bottom Yukawa couplings absorb the bulk of the SUSY-QCD corrections. After resummation the SUSY-QCD corrections cancel against the pure QCD corrections to a large extent. Thus, as in the top quark case the inclusion of both corrections is of vital importance. A comparison of the total resummed and unresummed NLO cross sections implies good agreement within $10 \%$ and thus a significant improvement of the perturbative stability from LO to NLO.

\section{Conclusions}

We have presented the full SUSY-QCD corrections to neutral MSSM Higgs radiation off top and bottom quarks at linear $e^{+} e^{-}$colliders. The size of the corrections is of $\mathscr{O}(10-20 \%)$ and of similar magnitude as the pure QCD corrections obtained in the past. This underlines the relevance of including these corrections in future analyses of these processes at linear $e^{+} e^{-}$colliders.

At large values of $\operatorname{tg} \beta$ Higgs radiation off bottom quarks provides a possibility to measure $\operatorname{tg} \beta$ [8]. In the past it has been demonstrated that the bulk of the pure QCD corrections can be absorbed in the running bottom Yukawa couplings, defined at the scale of the corresponding Higgs momentum flows [2]. We have shown that the resummation of nondecoupling contributions to the bottom Yukawa couplings reduce the SUSY-QCD corrections to a moderate size, similar to the pure QCD corrections in the resonant as well as continuum regimes [1].

\section{References}

[1] P. Häfliger and M. Spira, Nucl. Phys. B719 (2005) 35 [arXiv:hep-ph/0501164].

[2] S. Dittmaier, M. Krämer, Y. Liao, M. Spira and P.M. Zerwas, Phys. Lett. B478 (2000) 247 [arXiv:hep-ph/0002035].

[3] L. Hall, R. Rattazzi and U. Sarid, Phys. Rev. D50 (1994) 7048 [arXiv:hep-ph/9306309]; R. Hempfling, Phys. Rev. D49 (1994) 6168; M. Carena, M. Olechowski, S. Pokorski and C.E.M. Wagner, Nucl. Phys. B426 (1994) 269 [arXiv:hep-ph/9402253]; D. Pierce, J. Bagger, K. Matchev and R. Zhang, Nucl. Phys. B491 (1997) 3 [arXiv:hep-ph/9606211].

[4] M. Carena, D. Garcia, U. Nierste and C.E.M. Wagner, Nucl. Phys. B577 (2000) 88 [arXiv:hep-ph/9912516];

[5] J. Guasch, P. Häfliger and M. Spira, Phys. Rev. D68 (2003) 115001 [arXiv:hep-ph/0305101].

[6] B. C. Allanach et al., in Proc. of the APS/DPF/DPB Summer Study on the Future of Particle Physics (Snowmass 2001) ed. N. Graf, Eur. Phys. J. C 25 (2002) 113 [eConf C010630 (2001) P125] [arXiv:hep-ph/0202233].

[7] S. Dittmaier, M. Krämer, Y. Liao, M. Spira and P.M. Zerwas, Phys. Lett. B441 (1998) 383 [arXiv:hep-ph/9808433].

[8] For a recent summary see J.F.Gunion, T.Han, J.Jiang and A.Sopczak, Contribution to the LHC/LC Physics Document, eds. G.Weiglein et al., arXiv:hep-ph/0410364. 